\title{
A CRIAÇÃO DE UM QUADRO DE RESPONSABILIDADE SOBRE O DESENVOLVIMENTO NANOTECNOLÓGICO DA ARGENTINA
}

\section{CREATING A RESPONSIBILITY FRAMEWORK ON NANOTECHNOLOGICAL DEVELOPMENT IN ARGENTINA}

\section{Daniel Francisco Nagao Menezes ${ }^{1}$}

Resumo: As inovações tecnológicas são apresentadas com grandes expectativas de benefícios futuros e riscos difusos. No que diz respeito às inovações relacionadas à nanotecnologia, há um alto grau de incerteza sobre se as nanopartículas presentes nos produtos de consumo causam riscos à sociedade, por meio da saúde e do meio ambiente. Por isso é necessário incorporar um quadro de responsabilidade para o seu desenvolvimento. $\mathrm{O}$ objetivo deste artigo é fazer uma proposta para incorporar um marco de responsabilidade para o desenvolvimento da nanotecnologia na Argentina, oferecendo uma proposta concreta para responsabilizar o desenvolvimento da nanotecnologia na Argentina.

Palavras-chave: Marco de responsabilidade; Nanotecnologia; Inovação Tecnológica; Regulação da Inovação; Riscos.

Abstract: Technological innovations are presented with high expectations of future benefits and diffuse risks. With regard to innovations related to nanotechnology, there is a high degree of uncertainty about whether the nanoparticles present in consumer products cause risks to society, through health and the environment. That is why it is necessary to incorporate a framework of responsibility for its development. The purpose of this article is to make a proposal to incorporate a framework of responsibility for the development of nanotechnology in Argentina,

\footnotetext{
${ }^{1}$ Graduação em Direito (PUC-Campinas), Especializações em Direito Constitucional e Direito Processual Civil (PUC-Campinas), em Didática e Prática Pedagógica no Ensino Superior (Centro Universitário Padre Anchieta), Mestre e Doutor em Direito Político e Econômico (Universidade Presbiteriana Mackenzie), Pós-Doutor em Direito (USP). Pós-Doutorando em Economia (UNESP-Araraquara). Professor do Programa de Pós-Graduação em Direito Político e Econômico da Faculdade de Direito da Universidade Presbiteriana Mackenzie. Membro do CIRIECBrasil.
} 
offering a concrete proposal to make the development of nanotechnology in Argentina responsible.

Keywords: Responsibility framework; Nanotechnology; Technologic innovation; Regulation of Innovation; Scratch's.

\section{INTRODUÇÃO}

As inovações tecnológicas são apresentadas com grandes expectativas de benefícios futuros e riscos difusos. A compreensão deste problema na Europa levou à introdução da estrutura de Pesquisa e Inovação Responsável - Responsible Resarch and Innovation (RRI). O quadro RRI, que encontra os seus antecedentes no estudo das implicações e dos aspectos éticos, jurídicos e sociais (ELSI/ELSA), foi desenvolvido a partir da filosofia da ciência e se materializou no princípio da inclusão, que propõe a participação de todos os atores - ciência, política, indústria e sociedade civil - em todo o processo de inovação. Para isso, para além das questões éticas, de normalização e regulação, é imprescindível levar em consideração a articulação dos diferentes atores ligados ao desenvolvimento de uma tecnologia de ponta como a nanotecnologia.

A análise desta proposta europeia pode ser útil para o contexto local, mas não por importar ideias diretamente. Em vez disso, é necessário considerar as condições locais por meio de um processo de tradução contextualizado que torne visíveis as sugestões úteis para o país. No caso da nanotecnologia, por se encontrar em um estágio inicial de desenvolvimento, as ações promovidas pelo setor público devem ser reconsideradas de forma a incorporar um quadro de responsabilidade que integre e articule questões relacionadas à ética, padronização e regulação da esta tecnologia. Nesse sentido, o ponto de partida para se poder incorporar um quadro de responsabilidade pelo desenvolvimento da nanotecnologia a nível local consiste na descrição e 
análise das organizações existentes no país cujas áreas de interesse estão ligadas a questões relacionadas com a ética científica, padronização e regulamentação.

Com base nesta análise, e levando em consideração a viabilidade da articulação entre os diferentes atores vinculados ao desenvolvimento da nanotecnologia, é possível fazer uma proposta para incorporar um marco de responsabilidade no desenvolvimento da nanotecnologia na Argentina, que se constitui como o objetivo deste artigo. Para completá-lo, na primeira seção, é descrito um arcabouço de responsabilidades relacionadas às questões éticas, padronização e regulação da nanotecnologia, alertando-se sobre a necessidade de considerar as condições locais para incorporá-lo no país vizinho. Na segunda seção, são analisadas as organizações que promoveram o debate em torno das discussões éticas, padronização e regulação relacionadas ao setor de nanotecnologia na Argentina. Finalmente, na terceira seção, é feita uma proposta concreta para promover um marco de responsabilidade da Fundação Argentina de Nanotecnologia. Desta forma, o artigo oferece uma proposta concreta para responsabilizar o desenvolvimento da nanotecnologia na Argentina.

\section{A NECESSIDADE DE UMA ESTRUTURA DE RESPONSABILIDADE EM CIÊNCIA ADAPTADA AO AMBIENTE LOCAL}

Nas sociedades modernas, a inovação como conceito interdisciplinar é uma ideia central das políticas públicas. Uma das características das inovações é que seus efeitos subsequentes na sociedade são incertos e, as inovações tecnológicas, em particular, são apresentadas como uma configuração segura imediata, com grandes expectativas de benefícios futuros e riscos difusos (Konrad, 2010; Konrad, Van Lente, Groves, Selin; 2016).

Levando em consideração a existência de um contrato social implícito entre cientistas e o resto da sociedade (Guston, 2000; Owen et al., 2013), os primeiros obtêm relativa liberdade para realizar suas tarefas e inovar, e os últimos proporcionam o financiamento necessário em troca da expectativa de crescimento, desenvolvimento e geração de valor. Essa divisão moral do trabalho permite que os cientistas pesquisem e desenvolvam, e que a indústria introduza todos os tipos de novas tecnologias na sociedade, enquanto estruturalmente carece de meios 
para responsabilizar-se pelos efeitos contraproducentes que poderiam ocorrer em tal transferência.

No que se refere à nanotecnologia, há um alto grau de incerteza sobre como e em que grau as nanopartículas presentes nos produtos de consumo causam riscos à sociedade, por meio da saúde e do meio ambiente. Desse fato, o campo da nanotoxicologia emergiu da academia (Donaldson, Stone, Tran, Kreyling, Borm; 2004; Grieger, Hansen, Baun, 2009; Maynard et al., 2006; Oberdörster, Oberdörster, Oberdörster, 2005).

Atendendo às categorias de risco manufaturado (invisível, difícil de controlar e conter, e que não conhece limites geográficos), a nanotecnologia tem se caracterizado como um risco moderno (Beck, 2011; Throne-Holst, 2012). Assim, para sua efetiva regulamentação, é necessário compreender o processo de co-constituição de risco por diferentes atores (ciência, política indústria e sociedade civil) que o desenvolvimento desta tecnologia acarreta.

Nesse cenário, a responsabilidade do setor público, da promoção à regulação, é fundamental. A compreensão da nanotecnologia como um risco co-constituído conduziu à introdução do quadro de Investigação e Inovação Responsável (RRI) para o desenvolvimento de políticas da União Europeia. O conceito de desenvolvimento responsável foi incluído nos programas de política de pesquisa em nanotecnologia (Comissão Europeia, 2004, 2005; Nanoscale Science, Engineering and Technology Subcom Committee, 2004; Roco, Williams, Alivisatos; 1999). Afirmaram que a nanotecnologia deve ser desenvolvida respeitando os princípios éticos e estudando cientificamente os potenciais riscos à saúde e ao meio ambiente, de forma a fornecer as normas necessárias à sua regulamentação. Para isso, seria necessário que o desenvolvimento da nanotecnologia considerasse duas categorias. Por um lado, aquele que considera as implicações no meio ambiente e na saúde. De outro, os aspectos éticos, legais e sociais que nele envolvem.

O framework RRI encontra seus antecedentes no estudo das implicações e aspectos éticos, legais e sociais (ELSI/ELSA) vinculados ao desenvolvimento responsável da inovação em geral (Zwart, Landeweerd, Van Rooij; 2014). Essas questões foram abordadas a partir da 
filosofia da ciência e se materializaram no princípio da inclusão, que propõe a participação de todos os atores - ciência, política, indústria e sociedade civil - em todo o processo de inovação.

Para isso, para além das questões éticas, de normalização e regulação, é imprescindível levar em conta a articulação dos diferentes atores ligados ao desenvolvimento de tecnologias de ponta como a nanotecnologia.

A ligação entre a responsabilidade nas inovações e a integração da sociedade como dimensões ao longo do processo, conduziu à área da Ciência e Sociedade no âmbito do $7^{\circ}$ Programa-Quadro Europeu de Investigação, a ser transformada na área da Ciência na Sociedade (Zadrozny, 2007). O objetivo dessa modificação era promover a participação pública estabelecendo um diálogo bilateral entre a ciência e a sociedade civil. Hoje, o Programa-Quadro Europeu de Pesquisa: Horizonte 2020, esta área leva o título Ciência com e para a Sociedade, e por RRI é entendido um processo no qual a sociedade em geral e a ciência (cientistas e inovadores) (Comissão Europeia, 2012; Owen, Macnaghten, Stilgoe; 2012; Von Schomberg, 2011). Com a RRI, busca contribuir para o bem-estar humano, entendendo que as partes interessadas compartilham um compromisso com objetivos socialmente desejáveis (Deblonde, 2016; Von Schomberg, 2016).

Essa busca por objetivos sociais acima dos objetivos econômicos faz com que a RRI se oponha às ideias centrais dos Sistemas Nacionais de Inovação (Freeman, 1987; Lundvall, 1992; Nelson, 1993).

A análise desta proposta europeia pode ser útil para o contexto local, mas não por importar ideias diretamente. O problema da importação de políticas públicas de outras regiões ao longo da história levou a transferências acríticas de marcos que produziram muitos danos em nossas sociedades.

$\mathrm{Na}$ América Latina em geral, no Brasil e na Argentina em particular, onde as políticas de ciência, tecnologia e inovação continuam a ser realizadas no âmbito do SNI (Chudnovsky, 1999; MINCyT, 2011; SECyT, 2006), a inclusão de um marco de responsabilidade é 
fundamental. Para isso, é necessário considerar as condições locais por meio de um processo de tradução contextualizado que permita tornar visíveis sugestões úteis para o país.

Como em outros países dependentes de ciência e tecnologia, a premissa local é que, se essas especificações não forem atendidas, as políticas de inovação podem excluir em vez de incluir. Nesse sentido, fala-se frequentemente de inovações com inclusão social (Arocena, Sutz; 2009) e de tecnologias sociais (Thomas, 2012), mas não se leva em conta uma extensão da agenda da política científica nos países latino-americanos para que a inclusão de uma estrutura de responsabilidade é eficaz (Vasen, 2016a; 2016b).

Mesmo tendo em conta que as medidas de inclusão social são um requisito para que os desenvolvimentos de inovação realizados no âmbito do SNI não se sejam excludentes, é necessário incorporar um quadro de responsabilidades relacionadas com o desenvolvimento da nanotecnologia para que os riscos associados que a mesma acarreta ao desenvolvimento não afetem a sociedade.

Nesse sentido, a criação de centros de excelência científica integrados à indústria está vinculada unicamente ao propósito de melhorar a competitividade internacional e promover o crescimento econômico. Chama a atenção nesse setor a falta de atenção às questões sociais, como a proteção dos consumidores e trabalhadores contra potenciais riscos, e a ausência de fomento à participação das organizações sociais nas decisões de políticas públicas que se referem à nanotecnologia (Foladori, Figueroa, Edgard, Invernizzi, 2012).

No caso da nanotecnologia, por se encontrar em um estágio inicial de desenvolvimento, as ações promovidas pelo setor público devem ser reconsideradas de forma a incorporar um quadro de responsabilidade que integre e articule questões relacionadas à ética, padronização e regulação desta tecnologia. Realizar o desenvolvimento de tecnologias de ponta num quadro de responsabilidade significa incluir ativamente os cidadãos em todo o processo de inovação, desde a sua promoção à sua regulamentação. Para isso, é preciso haver organizações que possibilitem a criação de espaços de participação da sociedade. Desde a designação da nanotecnologia como área prioritária das políticas públicas no país, o Estado tem realizado diversas iniciativas, por um lado, no que se refere ao financiamento, e, por outro, no que diz 
respeito à criação de organizações que promovam o debate em torno de discussões éticas, padronização e regulamentação.

\section{UM MARCO DE RESPONSABILIDADE PÚBLICA PARA O} DESENVOLVIMENTO DA NANOTECNOLOGIA NA ARGENTINA

O ponto de partida para poder incorporar um quadro de responsabilidade para o desenvolvimento da nanotecnologia a nível local consiste na atividade dos organismos públicos existentes no país cujas áreas de interesse estão ligadas a temas relacionados com a ética científica, normalização e regulamentações que têm ou tiveram alguma conexão com o desenvolvimento da nanotecnologia no país. Analisa-se a atividade da Comissão Nacional de Ética em Ciência e Tecnologia (CECTE), do Instituto Argentino de Normalização e Certificação (IRAM), da Administração Nacional de Medicamentos, Alimentos e Tecnologia Médica (ANMAT), da Superintendência de Riscos Trabalhistas. (SRT) e o Serviço Nacional de Saúde e Qualidade Alimentar (SENASA).

Tabela 1. Resumo das agências e atividades

\begin{tabular}{|c|c|}
\hline $\begin{array}{l}\text { Órgãos públicos de ética, padronização } \\
\text { e regulamentação }\end{array}$ & $\begin{array}{l}\text { Criação de espaços de participação da } \\
\text { sociedade }\end{array}$ \\
\hline $\begin{array}{l}\text { Comitê Nacional de Ética em Ciência e } \\
\text { Tecnologia }\end{array}$ & $\begin{array}{l}\text { Promove uma instância de argumentação } \\
\text { crítica e transdisciplinar na avaliação de } \\
\text { projetos e leis, elaborando códigos de } \\
\text { conduta ética na investigação em } \\
\text { nanotecnologia. }\end{array}$ \\
\hline $\begin{array}{l}\text { Instituto Argentino de Normalização e } \\
\text { Certificação }\end{array}$ & $\begin{array}{l}\text { Desenvolve os padrões na área de } \\
\text { tecnologias que são desenvolvidas em } \\
\text { escala nanométrica. }\end{array}$ \\
\hline $\begin{array}{l}\text { Administração } \text { Nacional de } \\
\text { Medicamentos, Alimentos e Tecnologia } \\
\text { Médica }\end{array}$ & $\begin{array}{l}\text { Guias de avaliação de nanomedicina e } \\
\text { nanodispositivos: Observatório de } \\
\text { problemas sociais }\end{array}$ \\
\hline Superintendência de Riscos Trabalhistas & $\begin{array}{l}\text { Observatório de Nanotecnologia e Saúde } \\
\text { do Trabalhador. }\end{array}$ \\
\hline
\end{tabular}




\begin{tabular}{|l|l|}
\hline $\begin{array}{l}\text { Serviço Nacional de Saúde e Qualidade } \\
\text { Alimentar }\end{array}$ & $\begin{array}{l}\text { Interação com equipes de pesquisa em } \\
\text { nanociências e nanotecnologia. }\end{array}$ \\
\hline
\end{tabular}

Fonte: Elaboração própria

\subsection{Comité Nacional de Ética en la Ciencia y la Tecnología (CECTE)}

Levando em consideração as considerações éticas vinculadas ao desenvolvimento de tecnologia, em abril de 2004, o CECTE foi criado por meio da Resolução 004/2001 da Secretaria de Ciência, Tecnologia e Inovação Produtiva e confirmado pelas Resoluções 031/2002 e 600/2004. A sua função é analisar os problemas associados a este tema em todos os campos de investigação, que dizem respeito tanto ao trabalho de investigadores e instituições de investigação, como à formação de futuros cientistas. Ao CECTE cabe a responsabilidade pelo âmbito ético na produção do conhecimento científico, sua gestão, interpretação e divulgação dos resultados alcançados. Para isso, avalia projetos de políticas, leis e regulamentos que envolvem a pesquisa científica e as novas tecnologias nesta perspectiva, promovendo uma instância de argumentação crítica e transdisciplinar sobre problemas relevantes para a integridade e o progresso da ciência.

No que diz respeito ao desenvolvimento da nanotecnologia, o CECTE alertou desde o início para a necessidade de iniciar um debate para discutir a questão emergente da ética neste campo. Nesse sentido, ao mesmo tempo que leva em consideração os possíveis benefícios e consequências arriscadas de seu desenvolvimento, cujas implicações sociais devem ser antecipadas e analisadas, também reconheceu que a constituição da Fundação Argentina de Nanotecnologia (FAN) significou uma importante contribuição para a análise das relações entre ciência, sociedade e Estado (CECTE, 2005). Neste sentido, a intervenção mais concreta do CECTE no domínio da nanotecnologia tem sido a sua participação e desenvolvimento, em conjunto com os profissionais da especialidade, dos princípios éticos das suas práticas, na detecção dos valores fundamentais e na criação de códigos e comitês éticos.

\subsection{Instituto Argentino de Normalización y Certificación (IRAM)}


O IRAM é uma associação civil sem fins lucrativos, que foi fundada em 1935 por representantes dos vários setores da economia, governo e instituições científicas e técnicas. No campo da normalização, o IRAM é o único representante da Argentina perante a Organização Internacional de Normalização (ISO) e dirige os comitês técnicos nacionais que analisam os documentos em estudo, encaminha as propostas nacionais e define a posição da Argentina perante aquele órgão internacional.

Em 2008, o IRAM criou um comitê de nanotecnologia formado por especialistas locais de várias disciplinas e, como membro observador, acompanha os avanços na padronização técnica da ISO/TC 229. A principal função desse comitê é estabelecer políticas e linhas de ação para o desenvolvimento de padronização nesta área específica. Por sua vez, a função do subcomitê de nanotecnologias é desenvolver padrões no campo das tecnologias que são desenvolvidas em escala nanométrica (IRAM, 2009).

\subsection{Administración Nacional de Medicamentos, Alimentos y Tecnología Médica (ANMAT)}

A ANMAT é um órgão descentralizado da Administração Pública Nacional, criado em agosto de 1992, pelo decreto 1490/92. A sua função é colaborar na proteção da saúde humana, garantindo que os medicamentos, alimentos e dispositivos médicos à disposição dos cidadãos sejam eficazes - no sentido de cumprirem o seu objetivo terapêutico, nutricional ou diagnóstico declarado -, segurança - expressa em elevada relação benefício/risco e qualidade -respondo às necessidades e expectativas da população. Para isso, cabe a realização dos processos de autorização, registro, padronização, fiscalização e fiscalização dos produtos de sua competência em todo o território nacional. A ANMAT depende técnica e cientificamente das normas e diretrizes emanadas da Secretaria de Políticas, Regulação e Institutos do Ministério da Saúde, em regime de autarquia econômica e financeira.

A ANMAT identificou a nanotecnologia como uma questão estratégica para a administração nacional e em reunião com a FAN concordou em criar um grupo de trabalho com o objetivo de desenvolver uma série de guias de avaliação de nanomedicina e nanodispositivos (ANMAT, 2010). 
Desde a criação do observatório da ANMAT até ao dispositivo 907/2011 (ANMAT, 2011), pretendeu-se articular o órgão governamental com as diferentes instituições de saúde e cidadãs. Buscou-se identificar problemas sociais que norteiam o órgão regulador por meio de um sistema participativo e integrador.

\subsection{Superintendência de Riscos Laborais (SRT)}

Por sua vez, a SRT é um órgão dependente da Secretaria de Previdência Social do Ministério do Trabalho, Emprego e Previdência Social da Nação e foi criada em 1996 por meio da Lei $n^{\circ}$ 24.557. Tem como objetivo garantir o efetivo cumprimento do direito à saúde e segurança da população durante o período de trabalho, centralizando a sua atividade na concretização de empregos dignos, que preservem a saúde e segurança dos trabalhadores, promovendo uma cultura de prevenção. Além disso, a SRT colabora com os compromissos assumidos pelo Ministério do Trabalho, Emprego e Segurança Social da Nação e pelos estados provinciais no que se refere à erradicação do trabalho infantil, à regularização do emprego e ao combate ao trabalho não registado.

Com base na necessidade de proteger os trabalhadores dos riscos nanotecnológicos a que estão expostos, foi criado em 2014 o Observatório SRT de Nanotecnologia e Saúde do Trabalhador. Seu objetivo é a difusão de informação para a prevenção no uso destes materiais, seus novos usos, avanços em saúde e segurança, aplicações e espaços produtivos na Argentina, dados estatísticos e recomendações para trabalho seguro.

\subsection{Servicio Nacional de Sanidad y Calidad Agroalimentaria (SENASA)}

O SENASA é um órgão descentralizado, com autarquia econômico-financeira e técnicoadministrativa e personalidade jurídica própria, que pertence à órbita do Ministério da Agroindústria da Nação e é responsável pela execução das políticas nacionais de saúde e qualidade animal e segurança vegetal e alimentar de sua competência, bem como verificar o cumprimento da regulamentação em vigor sobre a matéria. Também é responsável pelo controle do trânsito federal e das importações e exportações de produtos, subprodutos e derivados de 
origem animal e vegetal, agroalimentares, farmacêutico-veterinário, agroquímicos, fertilizantes e aditivos.

No que diz respeito à nanotecnologia, o SENASA realizou em 2015 um seminário participativo sobre sua introdução no agronegócio e agroalimentação. Contou com a participação de diversas organizações reunidas com o objetivo de criar, fortalecer e articular capacidades para o desenvolvimento de nanotecnologias voltadas para cadeias de valor agroalimentares, além da formação e especialização de profissionais da região. A agenda de trabalho incluiu aspectos gerais da nanotecnologia; nanomateriais e caracterização; suas diferentes aplicações em saúde animal e proteção de plantas e abordagens regulatórias em nanotecnologia e alimentos. Dessa forma, o SENASA cumpre seu propósito de vincular seu corpo técnico a essas novas disciplinas e suas diferentes aplicações, por meio da interação com equipes de pesquisa em nanociências e nanotecnologia que permite identificar linhas de trabalho conjuntas e realizar novos projetos.

\section{PROPOSTA PARA PROMOVER UM QUADRO DE RESPONSABILIDADE PARA O DESENVOLVIMENTO DA NANOTECNOLOGIA}

$\mathrm{Na}$ seção seguinte, propõe-se incorporar um marco de responsabilidade no desenvolvimento da nanotecnologia na Argentina. Nesse sentido, é analisada a FAN e, tendo em vista que esta organização se propõe como o local adequado para promovê-la, retomando iniciativas conjuntas com o CECTE, IRAM, ANMAT, SRT e SENASA, com a participação do FAN como articulador dos diferentes organismos relacionados à nanotecnologia na Argentina.

A seguir descreve-se o surgimento da Fundação Argentina de Nanotecnologia (FAN), esta organização e, por suas características de órgão articulador, é proposta como o lugar adequado para promover um quadro de responsabilidade.

\subsection{Fundación Argentina de Nanotecnología}

A origem do FAN está associada às primeiras ações que o Estado realizou para promover um setor de nanotecnologia em nível local. Em 2004, o então Ministro da Economia 
da Nação, Roberto Lavagna, anunciou um importante programa de nanotecnologia que tinha como foco um único projeto patrocinado pela empresa americana Lucent Technologies (Lavagna, 2004). O projeto, que envolveu um investimento de US \$10.000.000, trouxe polêmica na comunidade científica e redefiniu as políticas de ciência, tecnologia e inovação em nanotecnologia (Andrini, Figueroa; 2008). Em decorrência dessa situação, por meio do decreto n 3 380/2005, o Ministério da Economia e Produção fica autorizado a instituir o FAN, situação também polêmica do ponto de vista ético da ciência (CECTE, 2005).

A partir de 2007, a FAN passou a estar sob a jurisdição do MINCyT, e atualmente desenvolve diversas atividades relacionadas com a divulgação e coordenação da nanotecnologia, como a organização de encontros que permitem o contato de cientistas, empresários e empresários, a fim de promover a transferência de conhecimento entre eles. Nesse sentido, cabe à FAN articular o setor de nanotecnologia por meio de diversas ações que contemplem diversos problemas. Em particular, vinculado aos riscos associados ao desenvolvimento da nanotecnologia, a FAN promoveu o programa Nanotecnologia e Sustentabilidade, que pretendeu desenvolver uma agenda de trabalho para a realização de atividades tendo em conta as ações, regulamentos e capacidades necessárias para torná-la sustentável no seu desenvolvimento. No mesmo sentido, o programa procurou detectar as capacidades da nanotecnologia para contribuir com os desafios da sustentabilidade ambiental, considerando que ambos os eixos são complementares e requerem a intervenção de vários atores (especialistas em nanotecnologia, funcionários, representantes de instituições de P\&D e empresas, entre outras) que serão responsáveis pela transferência de informações e conhecimentos para o restante da sociedade.

Embora atualmente a FAN continue ativa, não existe um programa que execute uma atividade regular ligada aos problemas sociais do desenvolvimento nanotecnológico.

$\mathrm{Na}$ Argentina, até agora, os programas de política não fazem referência explícita à incorporação de um marco de responsabilidade; no país, não se preocupa em incluir processos de articulação entre os diferentes atores para incorporar a sociedade ao longo do desenvolvimento de inovações. 
No entanto, a análise da adoção de um marco de responsabilidade na Argentina não passou despercebida às organizações mundiais. Uma avaliação positiva realizada pela OCDE (Working Party on Nanotechnology, 2013) é registrada como um precedente no assunto. O relatório menciona que o CECTE é o órgão responsável pela execução das políticas relacionadas ao desenvolvimento responsável da nanotecnologia no país. As Diretrizes para Conduta Responsável em Pesquisa em Nanociência e Nanotecnologia (Vila Seoane, 2011) integraram os temas de estudo do CECTE há alguns anos, mas atualmente sua pesquisa e percepção pública parecem ter saído de pauta e continua sendo uma área pouco explorada.

A iniciativa mais próxima do CECTE para promover o desenvolvimento responsável da nanotecnologia esteve ligada à adoção do Código de Conduta europeu, que foi rejeitado pela comunidade científica local e, atualmente, o desenvolvimento responsável da nanotecnologia não parece constituir um Prioridade do Comitê.

Durante a Conferência Internacional para Pesquisa Responsável em Nanociência e Nanotecnologia, organizada em 2008 pelo CECTE junto com FAN, o Centro ArgentinoBrasileiro de Nanociência e Nanotecnologia (CABNN), o Programa Argentino-Brasileiro de Ética em Ciência e Tecnologia (PABECyT), a Direção de Relações Internacionais do MINCyT e, patrocinada pela Direção Geral de Investigação da Comissão Europeia, levantou pela primeira vez questões éticas no uso e implementação da nanotecnologia na Argentina. Nesta reunião, o chefe da Unidade de Governança e Ética da Direção-Geral da Investigação da Comissão Europeia apresentou o Código de Conduta para a Investigação Responsável em Nanociências e Nanotecnologia (Comissão Europeia, 2009) e relatou os princípios e ações específicas destinadas a implementá-lo nos países membros da União. Em relação aos riscos, alertou sobre a segurança das nanopartículas e a necessidade de aplicar o princípio da precaução e implementar um código de conduta (Zilgalvis, 2008).

Embora na reunião tenha sido acordado que o CECTE, em conjunto com pesquisadores, industriais e membros da FAN, iniciaria o processo de elaboração de um código que contemplasse as diretrizes discutidas no MINCyT, essa proposta não avançou muito. Representantes do meio acadêmico, da indústria e de organizações governamentais da Argentina e do Brasil discutiram a possibilidade e conveniência de se adotar um código 
semelhante nos dois países, o que contribuiria para o avanço da nanotecnologia e poderia influenciar positivamente o intercâmbio entre a região e a União. Europeu. No entanto, tendo em vista que a inclusão do princípio da precaução deixava a decisão do que era produzido e do que não ficava nas mãos da Comissão Europeia, o que poderia prejudicar o desenvolvimento da nanotecnologia local, optou-se por não avançar no seu desenvolvimento e implantação no país.

O que existe e está declarado nos programas de políticas públicas (MINCyT, 2011; SECyT, 2006), são intenções de adoção de medidas específicas que garantam a inclusão social. $\mathrm{Na}$ área da nanotecnologia, por enquanto, persiste a tendência dos SNI's, chamando todas as instituições vinculadas ao seu desenvolvimento de Sistema Nacional de Nanotecnologia (Vila Seoane, 2011; 2014).

Para além desta iniciativa específica, todas as iniciativas e ações descritas até agora, embora tenham sido interessantes e tenham sido orientadas para o desenvolvimento da nanotecnologia num quadro de responsabilidade, não foram mantidas ao longo do tempo. Grande parte das ações promovidas pelas organizações relacionadas às questões de ética, padronização e regulamentação hoje são desarticuladas.

Por isso é importante retomar desta organização as diversas ações relacionadas à ética, padronização e regulação do desenvolvimento desta tecnologia de ponta. Tendo em conta que a FAN é a primeira, e possivelmente a principal, iniciativa local em termos de promoção e coordenação dos diferentes atores que trabalham na nanotecnologia.

\section{CONCLUSÕES}

$\mathrm{Na}$ Argentina, os programas de política relacionados ao desenvolvimento da nanotecnologia não consideram os riscos associados que esta tecnologia de ponta traz. Com base na análise das organizações vinculadas à articulação, questões éticas, padronização e regulação da nanotecnologia, o objetivo deste artigo foi fazer uma proposta para incorporar um quadro de responsabilidade no seu desenvolvimento. 
Na primeira seção, foi analisado o risco envolvido no desenvolvimento de inovações. No caso das inovações relacionadas à nanotecnologia, há um alto grau de incerteza sobre como e em que grau as nanopartículas presentes nos produtos de consumo causam riscos à sociedade, por meio da saúde e do meio ambiente. Foi referido que, com base neste problema, foi introduzido na Europa o quadro de Investigação e Inovação Responsável (RRI), um quadro que se desenvolveu a partir da filosofia da ciência e que se materializou no princípio da inclusão, que propõe a participação de todos os atores - ciência, política, indústria e sociedade civil - em todo o processo de inovação. Para tanto, para além das questões éticas, de normalização e regulação, para a sua incorporação é imprescindível ter em conta a articulação dos diferentes atores ligados ao desenvolvimento de tecnologias de ponta como a nanotecnologia.

A análise desta proposta europeia pode ser útil para o contexto local, mas não por importar ideias diretamente. Em vez disso, é necessário considerar as condições locais por meio de um processo de tradução contextualizado que torne visíveis sugestões úteis para o país. No caso da nanotecnologia, por se encontrar em um estágio inicial de desenvolvimento, as ações promovidas pelo setor público devem ser reconsideradas de forma a incorporar um quadro de responsabilidade que integre e articule questões relacionadas à ética, padronização e regulamentação desta tecnologia. Nesse sentido, na segunda seção, foram analisadas as organizações que fomentaram o debate em torno das discussões éticas, da padronização e da regulação relacionadas ao setor de nanotecnologia do país. Em particular, foi explicada a intervenção do CECTE no setor da nanotecnologia e mencionados os diversos organismos de normalização e regulação que têm ou tiveram alguma ligação com o desenvolvimento deste sector no país (IRAM, ANMAT, SRT e SENASA). .

Por fim, na terceira seção, foi descrita a origem do FAN. Esta organização foi definida como a responsável pela articulação dos diferentes atores vinculados ao desenvolvimento da nanotecnologia na Argentina, e se constatou que, embora atualmente ativa, não realiza nenhum programa que proponha atividades regulares relacionadas com o problema social do desenvolvimento nanotecnológico. Tendo em conta que a FAN é a primeira, e possivelmente a principal, iniciativa local em termos de promoção e articulação dos diferentes atores que atuam na nanotecnologia, a proposta deste artigo consistiu em promover um quadro de responsabilidade para o desenvolvimento de nanotecnologia desta organização, começando 
pela retomada das iniciativas realizadas em conjunto com o CECTE, IRAM, ANMAT, SRT e SENASA. Desta forma, o artigo ofereceu uma proposta concreta para responsabilizar o desenvolvimento da nanotecnologia na Argentina.

\section{REFERÊNCIAS}

ANDRINI, L.; FIGUEROA, S. El impulso gubernamental a las nanociencias y nanotecnologías en Argentina. in Foladori, G.; Invernizzi, N. Nanotecnologías en América Latina. Ciudad de México: Porrúa, 2008, pp. 33-48

ANMAT. Boletín para profesionales. Buenos Aires: Administración nacional de medicamentos alimentos y tecnología médica, 2010.

ANMAT. Disposición 907/2011: Observatorio ANMAT. Buenos Aires: Administración nacional de medicamentos alimentos y tecnología médica, 2011.

AROCENA, R.; SUTZ, J. Sistemas de innovación e inclusión social. Pensamiento Iberoamericano, vol. 5, n.2, pp. 99-120, 2009.

BECK, U. Sociedade de risco: Rumo a uma Outra Modernidade. São Paulo: Editora 34, 2011.

CECTE. Declaración sobre el Decreto 380/05. Buenos Aires: Comité Nacional de Ética en la Ciencia y la Tecnología, 2005.

CECTE. Anotaciones para una ética en la ciencia y la tecnología. El principio de precaución. Buenos Aires: Comité Nacional de Ética en la Ciencia y la Tecnología, 2010.

CECTE. Proposiciones para una ciencia y una tecnología socialmente responsables. Buenos Aires: Comité Nacional de Ética en la Ciencia y la Tecnología, 2014. 
CHUDNOVSKY, D. Políticas de ciencia y tecnología y el Sistema Nacional de Innovación en la Argentina. Revista de la CEPAL, vol. 67, pp. 153-171, 1999.

COMISSÃO EUROPÉIA. Hacia una estrategia europea para las nanotecnologías. Bruxelas: Comisión de las Comunidades Europeas, 2014.

COMISSÃO EUROPÉIA. Nanosciences and anotechnologies: An action plan for Europe 2005-2009. Bruxelas: Commission of the European Communities, 2005.

COMISSÃO EUROPÉIA. A code of conduct for responsible nanosciences and nanotechnologies research. Luxemburgo: Office for Official Publications of the European Communities, 2009.

COMISSÃO EUROPÉIA. Responsible Research and Innovation: Europe's ability to respond to societal challenges. Bruxelas: Commission of the European Communities, 2012.

DEBLONDE, M. RRI in the service of Sustainability. Apresentado em Responsible Research and Innovation (RRI): The Problematic Quest for Right Impacts, San Sebastián, 2016.

DONALDSON, K.; STONE, V.; TRAN, C. L.; KREYLING, W.; BORM, P. J. Nanotoxicology. Londres: BMJ Publishing, 2004.

FOLADORI, G.; FIGUEROA, S.; EDGARD, Z.-L.; INVERNIZZI, N. Características distintivas del desarrollo de las nanotecnologías en América Latina. Sociologias, vol. 14, n. 3 , pp. 330-363, 2012.

FREEMAN, C. Technology Policy and Economic Performance. Londres: Printer, 1987.

GRIEGER, K. D.; HANSEN, S. F.; BAUN, A. The known unknowns of nanomaterials: describing and characterizing uncertainty within environmental, health and safety risks. Nanotoxicology, vol. 3, n. 3, pp. 222-233, 2009. 
GUSTON, D. Retiring the social contract for science. Issues in science and technology, vol. 16, n. 4, pp. 32-36, 2000.

IRAM. Normas para una industria próspera y segura. Buenos Aires: Instituto argentino de normalización y certificación, 2009.

IRAM. Plan de estudio de normas 2015. Buenos Aires: Instituto argentino de normalización y certificación, 2015.

KONRAD, K. Governance of and by expectations. Paper apresentado no 2010 EASST Conference, September 2-4, 2010.

KONRAD, K.; VAN LENTE, H.; GROVES, C.; SELIN, C. Performing and Governing the Future in Science and Technology. in Felt, U.; Fouché, R.; Miller, C.; Smith-Doerr L. The Handbook of Science and Technology Studies. Massachusetts: MIT Press, 2016, pp. 465480.

LAVAGNA, R. El Gobierno impulsa el desarrollo de tecnología y la alfabetización digital. Apresentado no IDEA 40 Coloquio anual, Mar del Plata, 2004. Disponível em: http://www.ideared.org/coloquio40/sintesis/Lavagna_Inversiones.asp

LUNDVALL, B.-A. National innovation system: towards a theory of innovation and interactive learning. Londres: Pinter, 1992.

MAYNARD, A. D.; AITKEN, R. J.; BUTZ, T.; COLVIN, V.; DONALDSON, K.; OBERDÖRSTER, G. Safe handling of nanotechnology. Nature Publishing Group, vol. 444, pp. 267-269, 2006.

MINCyT. Argentina Innovadora 2020. Plan Nacional de Ciencia, Tecnología e Innovación: Lineamientos estratégicos 2012-2015. Buenos Aires: Ministerio de Ciencia, Tecnología e Innovación Productiva, 2011. 
NELSON, R. R. National innovation systems: a comparative analysis. Oxford: Oxford University Press, 1993.

OBERDÖRSTER, G.; OBERDÖRSTER, E.; OBERDÖRSTER, J. Nanotoxicology: An Emerging Discipline Evolving from Studies of Ultrafine Particles. Environm Health Perspec, vol. 113, n. 7, pp. 823-839, 2005.

OWEN, R.; MACNAGHTEN, P.; STILGOE, J. Responsible research and innovation: From science in society to science for society, with society. Science and Public Policy, vol. 39, n.6, pp. 751-760, 2012.

OWEN, R.; STILGOE, J.; MACNAGHTEN, P.; GORMAN, M.; FISHER, E.; GUSTON, D. A framework for responsible innovation. In Owen, R.; Bessant, J.; Heintz M. (orgs.), Responsible innovation: managing the responsible emergence of science and innovation in society. Chichester: Wiley, 2013, pp. 27-50.

ROCO, M. C.; WILLIAMS, R. S.; ALIVISATOS, P. Nanotechnology Research Directions. IWGN Workshop Report, 1999.

THOMAS, H. Tecnologías para la inclusión social en América Latina: de las tecnologías apropiadas a los sistemas tecnológicos sociales. Problemas conceptuales y soluciones estratégicas. In Thomas, H.; Fressoli, M.; Santos, G. Tecnología, desarrollo y democracia: nueve estudios sobre dinámicas socio-técnicas de exclusión/inclusión social. Buenos Aires: Ministerio de Ciencia, Tecnología e Innovación Productiva de la Nación, 2012, pp. 25-78.

THRONE-HOLST, H. Consumers, Nanotechnology and Responsibilities Operationalizing the Risk Society. Oslo: University of Twente, 2012.

VASEN, F. ¿Estamos ante un "giro poscompetitivo" en la política de ciencia, tecnología e innovación? Sociologias, vol. 18, n. 41, pp. 242-268, 2016. 
VASEN, F. Responsible Innovation in Developing Countries: an enlarged agenda. in Asveld, L. et al. (orgs.). Responsible innovation: a European agenda? New York: Springer, 2016, pp. 93-109.

VILA SEOANE, M. Nanotecnología: su desarrollo en Argentina, sus características y tendencias a nivel mundial (Tesis de Maestría Gestión de la Ciencia, la Tecnología y la Innovación). Buenos Aires: Universidad Nacional de General Sarmiento, 2011.

VILA SEOANE, M. Los desafíos de la nanotecnología para el desarrollo en Argentina. Mundo Nano. Revista Interdisciplinaria en Nanociencia y Nanotecnología, vol. 7, n. 13, pp. 78-94, 2014.

VON SCHOMBERG, R. Introduction: Towards responsible research and innovation in the information and communication technologies and security technologies fields. in Von Schomberg, R. (org.). Towards responsible research and innovation in the information and communication technologies and security technologies fields. Luxemburgo: Publications Office of the European Union, 2011, p. 07-15.

VON SCHOMBERG, R. Responsible Innovation for the Pursuit of Sustainability. Paper apresentado na Responsible Research and Innovation (RRI): The Problematic Quest for «Right» Impacts, San Sebastián, 2016.

ZILGALVIS, P. Recommendation to the Member States on a Code of Conduct for Responsible nanosciences and Nanotechnologies Research. Paper apresentado na Conferencia Internacional para la Investigación Responsable en Nanociencia y Nanotecnología, 2008. Disponível em: http://www.cecte.gov.ar/pdf/000057-es.pdf

ZWART, H.; LANDEWEERD, L.; VAN ROOIJ, A. Adapt or perish? Assessing the recent shift in the European research funding arena from 'ELSA' to 'RRI'. Life Sciences, Society and Policy, vol. 10, n. 1, pp. 01-19, 2014. 
Agradecimentos:

O presente trabalho foi realizado com apoio da Coordenação de Aperfeiçoamento de Pessoal de Nível Superior - Brasil (CAPES) - Código de Financiamento 001. Processo 88881.310388/2018-01 - PRINT / CAPES (Infraestrutura Legal da Nanotecnologia). 